\title{
Inhibidores de la tirosina-cinasa en cáncer de tiroides
}

\section{Tyrosine-kinase inhibitors for thyroid carcinoma}

\author{
Román-González $A^{1}$, Mejía S $S^{2}$ Zapata $M L^{2}$.
}

${ }^{1}$ Departamento de endocrinología y metabolismo, Hospital Universitario San Vicente Fundación-Universidad de Antioquia, Medellín, Colombia. Sección de endocrinología y diabetes, departamento de Medicina Interna. Profesor de Endocrinología, Universidad de Antioquia, Medellín, Colombia.

${ }^{2}$ Departamento de oncología, Hospital Universitario San Vicente Fundación, Medellín, Colombia. Profesor, Universidad de Antioquia.

Autor de correspondencia: Alejandro Román-González Correo electrónico: alejoroman@gmail.com

Fecha de recepción: 8/08/2019

Fecha de aceptación: 28/01/2020

\section{Resumen}

Introducción: el carcinoma de tiroides es la neoplasia endocrina más común. Su prevalencia está en aumento por varias causas, entre las cuales se encuentra el sobrediagnóstico. A pesar de lo anterior, también existe un incremento en los tumores más grandes, más agresivos y en la mortalidad que producen. A la fecha, la causa de esto es desconocida. La mayoría de los pacientes con cáncer de tiroides tienen enfermedad localizada. Sin embargo, el $15 \%$ de los pacientes puede tener enfermedad metastásica y hasta la mitad de ellos puede tener enfermedad resistente al yodo. Para estos pacientes, las opciones han sido pocas.

Metodología: se revisaron los estudios aleatorizados y controlados con placebo de los inhibidores de tirosina-cinasa en pacientes con carcinoma de tiroides y se presentó una síntesis de sus resultados, lo mismo que las indicaciones generales para al manejo de dicha enfermedad.

Resultados: a la fecha, el único medicamento aprobado en Colombia para usar en cáncer de tiroides metastásico o irresecable, resistente a yodo y en progresión es el sorafenib. Aprobados en otros países se encuentra el lenvatinib y la FDA recientemente aprobó la combinación dabrafenib más trametinib para pacientes BRAF mutados o con cáncer anaplásico BRAF mutado. El manejo de los efectos adversos de estos medicamentos es esencial para el éxito de la terapia. Se discute también la evidencia de tratamiento en carcinoma medular de tiroides, terapias futuras y el papel de la inmunoterapia.

Conclusiones: el cáncer de tiroides resistente a yodo es un reto clínico. Los inhibidores de la tirosina-cinasa son una opción para estos pacientes. El manejo de los efectos adversos es esencial para un uso adecuado y una terapia exitosa.

Palabras clave: proteínas proto-oncogénicas B-raf, neoplasias de la tiroides, lenvatinib, sorafenib, inhibidores de proteínas quinasas.

\section{Abstract}

Introduction: Thyroid carcinoma is the most common endocrine neoplasia. An increase in the prevalence of this disease has been found and maybe related to over-diagnosis. However, an increase in larger tumor, aggressive variants and mortality has also been found. The cause of this change if currently unknown. Most patients with thyroid cancer have localized, low-risk disease. Between 1 to $5 \%$ of thyroid carcinoma have metastasis. Half of them may have radioactive resistant tumor. Therapeutical options in these patients has been limited.

Methods: A literature review of randomized clinical trials versus placebo evaluating tyrosine-kinase inhibitors in thyroid carcinoma was performed. A synthesis of the results is presents was well as general indications for metastatic disease management.

Results: To the date of publication of this article, the only approved drug in Colombia to treat advanced thyroid carcinoma is sorafenib. In other countries, Lenvatinib has been approved. The combination of dabrafenib plus trametinib was recently approved by the FDA to be used in patients with BRAF mutations. An active management of side effects is required for a successful and safe treatment.

Conclusions: Radioactive resistant thyroid carcinoma is a clinical challenge. Tyrosine-kinase inhibitors are an option for these patients. Side effects management is essential for an appropriate and successful use.

Keywords: proto-oncogene proteins B-raf, thyroid neoplasms, lenvatinib, sorafenib, protein kinase inhibitors. 


\section{Introducción}

El cáncer de tiroides se clasifica en bien diferenciado, en los que se encuentran el carcinoma papilar y el folicular, ambos originados en las células foliculares, y en carcinoma pobremente diferenciado y anaplásico, entidades con mayor mortalidad y complejidad en su manejo. Otros tipos raros de tumores tiroideos son el linfoma, el adenoma trabecular hialinizante (1) y el paraganglioma.

El carcinoma papilar de tiroides (CPT) es una epidemia en crecimiento. Es el primer cáncer endocrino y corresponde al 2,1 \% de todo el cáncer en el mundo (2). Para el año 2020 se considera que será el tercer cáncer en frecuencia en mujeres en edad fértil, dado que el $77 \%$ de todo el cáncer de tiroides ocurre en mujeres. El número estimado de casos nuevos de cáncer de tiroides globalmente para el 2018 fue de 567.233 casos, 3,1\% de todo el cáncer y doceava causa de cáncer, y ocurrieron 41.071 muertes correspondientes al 0,4 \% de la mortalidad por cáncer en el mundo (3). A pesar de que la mayoría tiene un desenlace exitoso, con unas tasas elevadas de curación solo con el manejo quirúrgico, un porcentaje de estos casos tendrá recurrencias locorregionales y entre el $1 \%$ y el 5 $\%$ tendrá enfermedad metastásica. Afortunadamente, el $90 \%$ de los casos de cáncer de tiroides son diferenciados y, en términos generales, son curables con tratamiento quirúrgico.

Otro tipo muy interesante neoplasia es el carcinoma medular de tiroides (CMT). Este es un tumor neuroendocrino derivado de las células $\mathrm{C}$ y se clasifica como tumor tiroideo por su localización anatómica. Es infrecuente y se estima que corresponde, en términos generales, al 1 \%-2 \% de todo el cáncer de tiroides (4). Las células $\mathrm{C}$ parafoliculares de la tiroides provienen de la cresta neural y cumplen un papel mínimo en la fisiología del ser humano adulto, aunque son responsables de la secreción de calcitonina y, por ende, este es uno de los marcadores tumorales usados en el seguimiento del CMT.

Si bien los pacientes con CPT y CMT pueden ser curados con cirugía si el tumor está localizado, en algunos casos estos se presentan como enfermedad metastásica, sintomática y progresiva a pesar de los tratamientos estándar (4). En estos casos es necesario el uso de terapias sistémicas como los inhibidores de la tirosina-cinasa (ITK) (5). En esta revisión se discutirán las bases moleculares del CPT y del CMT, los principios oncológicos para el enfoque y manejo del paciente con cáncer avanzado de tiroides y la evidencia de los ITK aprobados en esta enfermedad (6-11), finalmente, se mencionarán brevemente las terapias adicionales.

\section{Carcinoma papilar}

El manejo de la enfermedad metastásica tiroidea papilar incluye el uso de yodo radioactivo, radioterapia y control regional con técnicas como la ablación con etanol o radiofrecuencia. Su uso está determinado por la tasa de progresión del tumor, la ca- pacidad de este para captar yodo radioactivo, la disponibilidad de técnicas de radiología intervencionista, el tamaño y número de metástasis, así como de los síntomas del paciente.

La minoría de los pacientes con carcinoma bien diferenciado de tiroides va a tener enfermedad metastásica con resistencia a la terapia con yodo radioactivo. Este grupo ha tenido pocas opciones terapéuticas ya que el uso de la única quimioterapia sistémica aprobada previa a los ITK, la doxorrubicina, tenía pésimos resultados oncológicos, con medianas de supervivencia a largo plazo del $10 \%$.

\section{Biología del CPT}

El factor más claramente implicado en la patogénesis de este cáncer es la radiación ionizante, como en el evento nuclear ocurrido en Chernóbil (26 de abril de 1986). Se ha postulado el aumento en la TSH y la tiroiditis de Hashimoto, así como condiciones familiares como la enfermedad de Cowden, el síndrome de Werner y la poliposis adenomatosa familiar. Se han documentado mutaciones en BRAF, RAS y rearreglos tipo RET/PTC; esta última es la más común en el carcinoma de tiroides asociado al accidente de Chernóbil (12). Por su parte, las mutaciones en RAS se han asociado más a carcinoma folicular de tiroides (12).

\section{Inhibidores de la tirosina-cinasa en CPT}

Las tirosina-cinasas son moléculas que funcionan como intermediarios de la señalización celular, estimulando su proliferación, angiogénesis, invasión, metástasis y autorregulación. De esta forma, los ITK actúan inhibiendo estas moléculas e interrumpiendo las vías implicadas en la proliferación celular y en la angiogénesis de las células tumorales. En la actualidad son una terapia dirigida aprobada para el tratamiento del cáncer diferenciado y pobremente diferenciado, resistente a yodo radioactivo y en progresión.

El efecto de estas moléculas se debe entender como un modulador del crecimiento tumoral o "tumor-estático", con el resultado principal de la estabilización de la enfermedad y el retardo en su progresión. En menor proporción tienen un efecto directo en la célula tumoral que lleva a su apoptosis y como resultado se disminuye la carga tumoral. Los resultados clínicos encontrados con los ITK resaltan la importancia de la vía antiangiogénica en el control del CPT metastásico y la independencia de su efecto de las mutaciones de vías intracelulares asociadas a enzimas tirosina-cinasa.

Para el uso de estas terapias el paciente debe cumplir, como mínimo, los siguientes requisitos:

- Cáncer de tiroides localmente avanzado irresecable o metastásico.

- Imposibilidad de realizar otras terapias como yodoterapia, radiofrecuencia, etanol, radioterapia o resección quirúrgica.

- Resistencia al yodo radioactivo. 
- Progresión de la enfermedad documentada mediante imagen diagnóstica con uso de RECIST.

- Tomografía de emisión de positrones positiva para tejido metabólicamente activo (aunque no es un criterio mandatorio en los ensayos clínicos, sería ideal).

- Estado funcional adecuado (ECOG 0-2 o índice Karnofsky $70 \%-100 \%)$.

La única terapia aprobada y disponible en nuestro medio a la fecha es el sorafenib. La otra terapia aprobada por la FDA es el lenvatinib que no está disponible en nuestro medio de manera rutinaria dado que requiere importación como vital no disponible. En estudios fase II se evalúan otras terapias moleculares como el everolimus, los inhibidores de BRAF (dabrafenib, vemurafenib) y los inhibidores de MEK, como trematinib y selumetinib, entre otros. El uso de estas terapias debe realizarse por un grupo multidisciplinario de tumores endocrinos con interés en cáncer de tiroides, con médicos nucleares, cirujanos de cabeza y cuello, oncólogos y endocrinólogos entrenados en el manejo del cáncer avanzado de tiroides y de los efectos adversos de los inhibidores de la tirosina-cinasa.

\section{Evidencia de los medicamentos disponibles para tratar la enfermedad resistente a yodo radioactivo en progresión en pacientes con cáncer papilar de tiroides}

\section{Sorafenib}

En el estudio Sorafenib in radioactive iodine-refractory, locally advanced or metastatic differentiated thyroid cancer: a randomised, double-blind, phase 3 trial $(6,13)$ se incluyeron 419 pacientes mayores de 18 años con cáncer de tiroides localmente avanzado o cáncer de tiroides diferenciado metastásico refractario a yodo (papilar, folicular, incluido de células de Hurthle y pobremente diferenciado), con progresión en los últimos 14 meses acorde con los criterios de RECIST, al menos una lesión medible por tomografía computarizada (TC) o resonancia magnética (RM), ECOG 0-2; con función renal, medular y hepática normal y TSH menor de 0,5 mIU/L. El cáncer de tiroides refractario a yodo se definió como:

- la presencia de al menos una lesión sin captación de yodo; o

- pacientes con tumores yodocaptantes y:

- progresión luego de un tratamiento con yodo radioactivo en los últimos 16 meses; o

- progresión luego de dos tratamientos con yodo recibidos con menos de 16 meses de diferencia (la última dosis recibida más de 16 meses); o

- una dosis acumulada de yodo igual o superior a 600 $\mathrm{mCi}$.

Se excluyeron los pacientes que habían recibido previamente terapia dirigida, talidomida o quimioterapia para cán- cer de tiroides, cirugía previa o trauma en los 30 días previos al inicio del medicamento, antecedente de cáncer de piel, cérvix o vejiga en los últimos 5 años, cáncer indiferenciado de tiroides, úlceras, infección activa o sangrado en los últimos 3 meses, historia de hemorragia, infiltración traqueal, bronquial o esofágica; cardiopatía o hipertensión arterial no controlada, infección por VIH o hepatitis, embarazo o lactancia y alergia al sorafenib. Se permitieron las dosis bajas de quimioterapia para radiosensibilización.

La intervención fue sorafenib en $400 \mathrm{mg}$, cada 12 horas $(n=209)$, hasta progresión radiológica, toxicidad inaceptable o muerte, comparado con placebo $(n=210)$. Los desenlaces primarios de este estudio fueron: supervivencia libre de progresión (cada 8 semanas) con criterios RECIST: 1 . Nuevas lesiones óseas; 2.20 \% o más de crecimiento; y 3. Necesidad de radioterapia. Los secundarios fueron: supervivencia global, tiempo hasta la progresión, tasa de respuesta objetiva (parcial o completa), tasa de control de la enfermedad (parcial, completa o estable $\geq 4$ semanas $/ 6$ meses) y duración de la respuesta. Se demostró que la supervivencia libre de progresión fue mayor en el grupo de sorafenib que en el grupo de placebo $(10,8$ meses vs. 5,8 meses; HR: 0,59; IC 95 \%: 0,45-0,76; $p<0,0001$ ). Al momento del cierre del seguimiento, la mediana fue de 16,2 meses; para ese momento 63 pacientes continuaban en tratamiento. Del grupo placebo que progresaron, el 71,4 \% recibió sorafenib, lo cual puede diluir el impacto en la supervivencia global. Luego del estudio 60 pacientes recibieron alguna terapia anticáncer y de aquellos pacientes que alcanzaban una respuesta parcial o completa se lograba un control a 6 meses en el $54 \%$. La dosis promedio de sorafenib fue de $651 \mathrm{mg}$. En análisis de subgrupos el beneficio no era significativo en lesiones tumorales menores de 1,5 centímetros, en pacientes con tumores foliculares o pobremente diferenciados y en pacientes con PET con 18F-FDG negativo. El grupo de sorafenib tuvo una mayor tasa de eventos adversos de cualquier grado $(98,6$ vs. 87,6\%); aquellos mayores de grado 3 se presentaron en un $37,2 \%$ (26,3 \% en el grupo placebo). La causa de suspensión del tratamiento más frecuente fue el síndrome de mano/pie en el 76,3\%. Otros efectos adversos fueron diarrea (68,6\%), alopecia $(67,1 \%)$, brote cutáneo $(50,2 \%)$, fatiga $(49,8 \%)$, pérdida de peso (46,9\%) e hipertensión (40,6 \%). Solo se reportó una muerte, que se relacionó con el tratamiento y fue causada por un evento coronario agudo. En conclusión, el sorafenib mejora significativamente la supervivencia libre de progresión comparado con placebo en pacientes con cáncer de tiroides diferenciado, en progresión y resistente a yodo radioactivo.

\section{Lenvatinib}

El estudio Lenvatinib versus Placebo in Radioiodine Refractory Thyroid Cáncer (SELECT) (7) incluyó pacientes mayores de 18 años con cáncer diferenciado de tiroides confirmado con patología, con lesiones medibles y evidencia de enfermedad 
refractaria al yodo. Las lesiones fueron definidas como al menos uno de los siguientes criterios: una lesión medible sin captación en cualquier rastreo con ${ }^{131}$ yodo; al menos una lesión medible que ha progresado acorde con el RECIST versión 1.1, dentro de los 12 meses después de terapia con ${ }^{131}$ yodo a pesar de la captación en cualquier momento del tratamiento; o actividad acumulada de ${ }^{131}$ yodo mayor de $600 \mathrm{mCi}$ y una revisión independiente de evidencia radiológica de progresión dentro de los 13 meses previos. Los pacientes elegibles no deberían haber recibido terapia previa con un ITK o un tratamiento previo con un solo régimen de ITK. La intervención fue lenvatinib oral en $24 \mathrm{mg}$, cada 24 horas, o placebo, en ciclos de 28 días, y el desenlace primario fue supervivencia libre de progresión. Esta se definió como el tiempo desde la aleatorización hasta el momento de la primera documentación de progresión de la enfermedad por revisión radiológica independiente o muerte. Se evaluó por intención de tratar.

Los desenlaces secundarios fueron: respuesta objetiva según RECIST versión 1.1. y supervivencia global. Las evaluaciones exploratorias de eficacia incluyeron tasa de control de la enfermedad y tasa de beneficio clínico También se evaluó la supervivencia libre de progresión.

El resultado principal fue una supervivencia libre de progresión de 18,3 meses en el grupo de lenvatinib y de 3,6 meses en el grupo placebo (HR para progresión o muerte: 0.21; IC 99 \%: 0,14-0,31; $p<0,001)$. El beneficio de supervivencia libre de progresión fue observado en todos los grupos preespecificados. La tasa de respuesta fue del 64,8 \% en lenvatinib (se lograron 4 respuestas completas y 165 respuestas parciales). La mediana de supervivencia global no fue alcanzada en los grupos. La dosis promedio recibida de lenvatinib fue de 17,2 mg. A pesar de las respuestas logradas, el control de la enfermedad a 6 meses fue solo del 15,3\%. En el subgrupo de pacientes que habían recibido ITK previo, el desempeño de lenvatinib se mantenía logrando una mediana de tiempo libre sin progresión de 15,1 meses. Respecto a la seguridad del medicamento, los efectos adversos de cualquier grado ocurrieron en el $97 \%$ de los pacientes. En el grupo de lenvatinib, los más comunes fueron: hipertensión $(67,8 \%)$, diarrea $(59,4 \%)$, fatiga o astenia (59,0\%), disminución del apetito (50,2\%), disminución de peso $(46,4 \%)$ y náusea $(41,0 \%)$. La suspensión del tratamiento por efectos adversos ocurrió en 37 pacientes con lenvatinib (14,2 \%) y 3 pacientes con placebo (2,3\%). En el grupo de lenvatinib, 6 de 20 muertes fueron consideradas estar relacionadas con el tratamiento.

\section{Carcinoma medular}

\section{Biología molecular del CMT}

El RET (del inglés re-arranged during transfection) es un protooncogen que codifica una proteína tirosina-cinasa. Está localizado en el cromosoma 10q11.2 e involucrado directamen- te en la patogénesis del CMT (14). Casi todos los pacientes con CMT hereditario (neoplasia endocrina múltiple tipo 2A y 2B) y hasta un $50 \%$ a $60 \%$ de los casos esporádicos presentan mutaciones en RET. Este gen fue descubierto por Takahashi en 1985 y está expresado en células derivadas de la cresta neural (15).

Las mutaciones en RET producen una activación constitutiva de la función cinasa de este receptor; por ende, las vías de señalización de este receptor se mantienen activas generando proliferación de las células C parafoliculares. La proteína RET tiene un dominio de unión a ligando extracelular compuesto de una región tipo cadherina y un dominio altamente conservado rico en cisteína. La región intracelular está compuesta de dos dominios tirosina-cinasa. La unión del ligando hace que el dominio rico en cisteína facilite la dimerización del receptor y produzca autofosforilación y activación de la tirosina-cinasa y de las vías de señalización, incluidas RAS/RAF/MAPK, PI3K/ AKT y JAK/STAT $(14,16)$.

Aunque no es claro cómo las mutaciones en RET producen carcinoma medular (17), estudios recientes señalan al ATF4 en la vía del RET como un actor implicado en la patogénesis del CMT. ATF4 es un factor de transcripción de la familia CREB, que desempeña un papel en la regulación de la respuesta al estrés. Este factor activa y aumenta la expresión de las proteínas NOXA y PUMA. Estas dos proteínas activan la vía de las caspasas y promueven la apoptosis. La activación permanente del RET mutado en CMT disminuye la transcripción de ATF4, estimula su ubiquitinación y subsecuente degradación. Por ende, causa regulación a la baja de NOXA y PUMA, lo que disminuye la apoptosis de las células (18).

Otra alteración importante en el CMT es el aumento en la expresión del factor de crecimiento derivado del endotelio vascular (VEGF), que influye en la capacidad de angiogénesis, invasión y metástasis de este tumor. Se han descrito también mutaciones en HRAS, KRAS, BRAF, KT, MLH1, STK11 y MET en baja frecuencia (17).

El tipo de mutación se correlaciona con el fenotipo clínico. Los pacientes con MEN2B, por la mutación M918T, tienen un curso clínico más agresivo y un peor pronóstico (19). Las mutaciones en RET se clasifican según su agresividad, basadas en el desarrollo de cáncer medular de tiroides a edades temprana y la asociación con enfermedad metastásica en tres categorías: muy alto riesgo, alto riesgo y riesgo moderado.

\section{Inhibidores de la tirosina-cinasa en CMT}

Aunque el CMT es infrecuente en la práctica clínica, enfrentarse a pacientes con este tipo de cáncer en estadios avanzando es un reto terapéutico, dado que son pacientes incurables con enfermedad progresiva asociada a manifestaciones graves, entre ellas diarrea de difícil manejo o síndrome de Cushing ectópico, con los efectos secundarios del hipercortisolismo como diabetes, hipopotasemia, miopatía, osteoporosis e infecciones $(20,21)$. Adicionalmente, en estadios avanzados, 
la quimioterapia y la radioterapia han sido consideras clásicamente de utilidad limitada $(22,23)$.

Actualmente se encuentran aprobados por la FDA dos medicamentos para el tratamiento del CMT: vandetanib y cabozantinib $(23,24)$. A continuación, se presenta la evidencia, los usos y las contraindicaciones de estos medicamentos.

\section{Vandetanib}

Es un medicamento oral que inhibe selectivamente al receptor del factor de crecimiento derivado del endotelio vascular (VEGFR-2 y 3), EGFR y a RET (25).

Evidencia aprobatoria para el uso de vandetanib. Estudio ZETA. Estudio fase III

En 2011 se publicó un estudio aleatorizado y comparado con placebo con vandetanib en pacientes con CMT (10). Se aleatorizaron 331 pacientes, 231 asignados a vandetanib y 100 asignados a placebo. Los pacientes elegibles fueron aquellos con CMT localmente avanzado no resecable o metastásico, bien fuera esporádico o hereditario, adecuada clase funcional (ECOG 0-2) y una calcitonina $\geq 500 \mathrm{pg} / \mathrm{mL}$. La dosis de inicio fue $300 \mathrm{mg} / \mathrm{d}$ hasta progresión de la enfermedad. El objetivo principal del estudio fue la supervivencia libre de progresión por una revisión central acorde con el RECIST 1.0. Los pacientes del estudio tenían enfermedad esporádica en la mayoría de los casos (88 \% en vandetanib y $95 \%$ en placebo). Esta enfermedad era metastásica en el 94 \% y $97 \%$, respectivamente.

La mediana de supervivencia libre de progresión fue de 19,3 meses en el grupo placebo y no se alcanzó en el grupo de vandetanib (HR: 0,46; IC 95: 0,31-0,69). Se predijo mediante métodos estadísticos que la mediana predicha para vandetanib era de 30,5 meses. El subgrupo de pacientes con mutación en RET M918T pareciera tener una respuesta más alta comparado con los pacientes negativos para esta mutación. A la fecha del estudio, no se observaron diferencias en la supervivencia global.

El $12 \%$ de los pacientes en vandetanib suspendieron el tratamiento por efectos adversos. Más pacientes en el grupo de vandetanib requirieron reducción en la dosis por eventos adversos y $8 \%$ de los pacientes tuvo prolongación del QTc. El 49,3\% de los pacientes necesitaron aumento en la dosis de levotiroxina. Los efectos adversos más comunes fueron diarrea, brote, náuseas e hipertensión. Los efectos adversos grado 3 o mayores fueron diarrea, hipertensión, prolongación de QTc, fatiga, disminución en el apetito, brote, astenia, disnea y dolor lumbar. Otros efectos adversos reportados en el $10 \%$ o más de la población tratada con vandetanib fueron disminución de peso, nasofaringitis, tos, dermatitis acneiforme, dolor abdominal, insomnio, piel seca, vómito, acné, cefalea y fatiga.

\section{Cabozantinib}

Es un inhibidor multicinasa de VEGFR1, VEGFR2, RET y c-MET (26). Está aprobado para el tratamiento del cáncer me- dular de tiroides y el carcinoma renal (26-28) y actualmente su utilidad en el tratamiento del feocromocitoma metastásico está siendo evaluada en un estudio del MD Anderson Cancer Center $(29,30)$.

Evidencia aprobatoria para el uso de cabozantinib. Estudio EXAM. Estudio fase III

En este ensayo se incluyeron 330 pacientes con CMT recurrente, metastásico o localmente avanzado irresecable. Se asignaron a cabozantinib en 140 mg o a placebo. Se encontró una mejoría significativa en la supervivencia libre de progresión comparado con placebo de 11,2 vs. 4 meses (HR: 0,28; IC 95 \%: 0,19-0,40). Se encontró una repuesta parcial en el $27 \%$ de los pacientes tratados con cabozantinib. Los pacientes presentaron eventos adversos comunes como diarrea, estomatitis, síndrome de eritrodisestesia palmoplantar, hipertensión y dolor abdominal. Como eventos adversos menos comunes, pero significativos, se encontraron formación de fístula y osteonecrosis de la mandíbula.

Como criterio de inclusión de los estudios de cabozantinib se requería progresión de la enfermedad; por ende, las diferencias en las curvas de supervivencia entre los estudios de vandetanib y cabozantinib pueden ser explicadas por este hecho.

\section{Otras terapias en CMT}

En estudios pequeños se han investigado otros ITK como axitinib (31), sorafenib (32), sunitinib (33), pazopanib (34) y motesanib $(35,36)$ con respuestas parciales o estabilización de la enfermedad, aunque no se ha avanzado en su desarrollo clínico.

\section{Terapias futuras en cáncer de tiroides}

\section{Larotrectinib}

Los genes NTRK1, 2 y 3 codifican los receptores neurotróficos de tirosina-cinasa TRKA, B y C, respectivamente $(37,38)$. TRK está involucrada en el crecimiento, la diferenciación y la supervivencia de las neuronas. Las fusiones génicas oncogénicas en NTRK generan un TRK mutado que resulta en una activación constitutiva, independiente del ligando. Esta alteración está involucrada en varios tumores sólidos, incluido el cáncer de tiroides, en particular el CPT pediátrico $(39,40)$.

Larotrectinib es un inhibidor de TRK aprobado en 2018 para pacientes con tumores sólidos con fusiones génicas en NTRK incluido CPT $(37,39-44)$. Este fue un estudio con diseño tipo cesta (basket) en el que se incluyeron pacientes con diferentes tipos de tumores, pero con fusiones génicas en NTRK. En el estudio aprobatorio se incluyeron $9 \%$ de los pacientes con carcinoma de tiroides (41).

\section{BLU 667}

El protooncogen RET, aparte de estar mutado en CMT, también está activado en múltiples tipos de cáncer, como el carci- 
noma pulmonar no microcítico, y en el CPT (45). BLU-667 es un inhibidor selectivo potente de RET y es diez veces más potente para inhibir este protooncogen que los inhibidores multicinasa aprobados $(9,28,46,47)$. En estudios in vitro e in vivo se ha demostrado su potencia y en estudios iniciales clínicos en pacientes con cáncer pulmonar y CMT induce respuestas clínicas durables con mínima toxicidad $(48,49)$.

\section{Combinaciones}

\section{Dabrafenib más trametinib}

Un gen mutado comúnmente en CPT es BRAF. Una alternativa para el tratamiento de esta enfermedad es inhibir el BRAF mutado (50). La mutación más común es la V600E. Sin embargo, se puede generar resistencia, la cual es mediada en parte por MEK1. Dabrafenib inhibe a BRAF y trametinib bloquea a MEK e inhibe la resistencia a las terapias dirigidas a BRAF (51). La combinación de estas dos terapias está aprobada para melanomas BRAF mutados y cáncer de pulmón de células no pequeñas BRAF mutado $(52,53)$. Esta combinación fue aprobada en 2018 para cáncer anaplásico de tiroides BRAF mutado basado en un estudio que incluyó 23 pacientes con tasa de respuesta global del $69 \%$ (54).

\section{Inmunoterapia}

El carcinoma diferenciado de tiroides se considera un tumor con baja inmunogenicidad dada su baja carga tumoral. Sin embargo, esta característica no se puede generalizar para todos los tumores diferenciados. Si bien solo el $1 \%$ de los carcinomas diferenciados se asocia a inestabilidad microsatelital $(55,56)$, la respuesta a pembrolizumab, independientemen- te de la expresión de PDL1, lo hace una alternativa adecuada con respuestas que se pueden prolongar hasta los 18 meses (57). En ausencia de inestabilidad microsatelital, la expresión de PDL1 en carcinoma diferenciado de tiroides es baja y los resultados en cuanto a respuesta y control de la enfermedad sugieren que la estrategia más plausible sea la combinación (con otros agentes de inmunoterapia o terapias blanco) para lograr resultados favorables a largo plazo $(58,59)$.

\section{Conflictos de interés}

Alejandro Román-González ha recibido honorarios como conferencistas o patrocinio para participación en congresos médicos de las siguientes empresas o compañías: Sanofi, Amgen, Novartis, Ultragenix, Biosidius, Asociación Colombiana de Osteoporosis y Metabolismo Mineral, Asociación Colombiana de Endocrinología, Diabetes y Metabolismo, Universidad del Bosque, Valentech, Colciencias, Hospital Universitario San Vicente Fundación, Universidad de Antioquia, Baxalta y Novo Nordisk.

Sergio Mejía: conferencista: AstraZeneca, BMS, MSD, Novartis, Advisory Board: AstraZeneca y BMS. Intereses institucionales e investigación: Roche y BMS. Patrocinio para participación en congresos médicos: Amgen, BMS, Janssen, Merck, MSD y Roche. Asociación Colombiana de Hematología y Oncología (ACHO).

Maycos Leandro Zapata: conferencista: Abbott, AstraZeneca, BMS, MSD, Novartis, Roche y Sanofi. Advisory: AstraZeneca y BMS. Intereses institucionales e investigación: Roche y BMS. Patrocinio para participación en congresos médicos: Abbott, Amgen, BMS, Janssen, Merck, MSD y Roche. Asociación Colombiana de Hematología y Oncología (ACHO).

\section{Referencias}

1. Román-González A, Simón-Duque C, Camilo-Pérez J, Vélez-Hoyo A. [Trabecular hyalinizing adenoma of the thyroid (HAT): A report of two cases]. Gac Med Mex. 2016;152(1):111-5.

2. Cabanillas ME, McFadden DG, Durante C. Thyroid cancer. Lancet. 2016;388(10061):2783-95. doi: 10.1016/S0140-6736(16)30172-6

3. Bray F, Ferlay J, Soerjomataram I, Siegel RL, Torre LA, Jemal A. Global cancer statistics 2018: GLOBOCAN estimates of incidence and mortality worldwide for 36 cancers in 185 countries. CA Cancer J Clin. 2018;68(6):394424. doi: 10.3322 /caac. 21492

4. Galofré JC, Santamaría-Sandi J, Capdevila J, Navarro-González E, ZafónLlopis C, Ramón Y Cajal Asensio T, et al. Consensus on the management of advanced medullary thyroid carcinoma on behalf of the Working Group of Thyroid Cancer of the Spanish Society of Endocrinology (SEEN) and the Spanish Task Force Group for Orphan and Infrequent Tumors (GETHI) Endocrinol Nutr. 2015;62(4):e37-46. doi: 10.1016/j.endonu.2015.01.005

5. Spitzweg C, Morris JC, Bible KC. New drugs for medullary thyroid cancer: new promises? Endocr Relat Cancer. 2016;23(6):R287-97. doi: 10.1530/ ERC-16-0104

6. Brose MS, Nutting CM, Jarzab B, Elisei R, Siena S, Bastholt L, et al. Sorafenib in radioactive iodine-refractory, locally advanced or metastatic differentiated thyroid cancer: a randomised, double-blind, phase 3 trial. Lancet. 2014;384(9940):319-28. doi: 10.1016/S0140-6736(14)60421-9

7. Schlumberger M, Tahara M, Wirth LJ, Robinson B, Brose MS, Elisei R, et al. Lenvatinib versus placebo in radioiodine-refractory thyroid cancer. N Engl J Med. 2015;372(7):621-30. doi: 10.1056/NEJMoa1406470
8. Leboulleux S, Bastholt L, Krause T, de la Fouchardiere C, Tennvall J, Awada A, et al. Vandetanib in locally advanced or metastatic differentiated thyroid cancer: a randomised, double-blind, phase 2 trial. Lancet Oncol. 2012;13(9):897-905. doi: 10.1016/S1470-2045(12)70335-2

9. Thornton K, Kim G, Maher VE, Chattopadhyay S, Tang S, Moon YJ, et al. Vandetanib for the treatment of symptomatic or progressive medullary thyroid cancer in patients with unresectable locally advanced or metastatic disease: U.S. Food and Drug Administration drug approval summary. Clin Cancer Res. 2012;18(14):3722-30. doi: 10.1158/1078-0432.CCR-120411

10. Wells SA, Robinson BG, Gagel RF, Dralle H, Fagin JA, Santoro M, et al. Vandetanib in patients with locally advanced or metastatic medullary thyroid cancer: a randomized, double-blind phase III trial. J Clin Oncol. 2012;30(2):134-41. doi: 10.1200/JC0.2011.35.5040

11. Hoy SM. Cabozantinib: a review of its use in patients with medullary thyroid cancer. Drugs. 2014;74(12):1435-44. doi: 10.1007/s40265-014$0265-x$

12. Haroon Al Rasheed MR, Xu B. Molecular Alterations in Thyroid Carcinoma. Surg Pathol Clin. 2019;12(4):921-30. doi: 10.1016/j.path.2019.08.002

13. Brose MS, Cabanillas ME, Cohen EE, Wirth LJ, Riehl T, Yue H, et al. Vemurafenib in patients with BRAF(V600E)-positive metastatic or unresectable papillary thyroid cancer refractory to radioactive iodine: a non-randomised, multicentre, open-label, phase 2 trial. Lancet Oncol. 2016;17(9):1272-82. doi: 10.1016/S1470-2045(16)30166-8

14. Jhiang SM. The RET proto-oncogene in human cancers. Oncogene. 
2000;19(49):5590-7. doi: 10.1038/sj.onc.1203857

15. Takahashi M, Ritz J, Cooper GM. Activation of a novel human transforming gene, ret, by DNA rearrangement. Cell. 1985;42(2):581-8. doi: 10.1016/0092-8674(85)90115-1

16. Drilon A, Hu ZI, Lai GGY, Tan DSW. Targeting RET-driven cancers: lessons from evolving preclinical and clinical landscapes. Nat Rev Clin Oncol. 2018;15(3):151-67. doi: 10.1038/nrclinonc.2017.175

17. Ji JH, Oh YL, Hong M, Yun JW, Lee HW, Kim D, et al. Identification of Driving ALK Fusion Genes and Genomic Landscape of Medullary Thyroid Cancer. PLoS Genet. 2015;11(8):e1005467. doi: 10.1371/journal.pgen.1005467

18. Bagheri-Yarmand R, Williams MD, Grubbs EG, Gagel RF. ATF4 Targets RET for Degradation and Is a Candidate Tumor Suppressor Gene in Medullary Thyroid Cancer. J Clin Endocrinol Metab. 2017;102(3):933-41. doi: $10.1210 /$ jc. $2016-2878$

19. Wells SA, Asa SL, Dralle H, Elisei R, Evans DB, Gagel RF, et al. Revised American Thyroid Association guidelines for the management of medullary thyroid carcinoma. Thyroid. 2015;25(6):567-610. doi: 10.1089/ thy.2014.0335

20. Barroso-Sousa R, Lerario AM, Evangelista J, Papadia C, Lourenço DM, Lin $\mathrm{CS}$, et al. Complete resolution of hypercortisolism with sorafenib in a patient with advanced medullary thyroid carcinoma and ectopic ACTH (adrenocorticotropic hormone) syndrome. Thyroid. 2014;24(6):1062-6. doi: $10.1089 /$ thy.2013.0571

21. Baudry C, Paepegaey AC, Groussin L. Reversal of Cushing's syndrome by vandetanib in medullary thyroid carcinoma. $N$ Engl J Med. 2013;369(6):584-6. doi: 10.1056/NEJMc1301428

22. Hadoux J, Pacini F, Tuttle RM, Schlumberger M. Management of advanced medullary thyroid cancer. Lancet Diabetes Endocrinol. 2016;4(1):64-71. doi: 10.1016/S2213-8587(15)00337-X

23. Dadu R, Hu MN, Grubbs EG, Gagel RF. Use of Tyrosine Kinase Inhibitors for Treatment of Medullary Thyroid Carcinoma. Recent Results Cancer Res. 2015;204:227-49. doi: 10.1007/978-3-319-22542-5_11

24. Frampton JE. Vandetanib: in medullary thyroid cancer. Drugs. 2012;72(10):1423-36. doi: 10.2165/11209300-000000000-00000

25. Gómez K, Varghese J, Jiménez C. Medullary thyroid carcinoma: molecular signaling pathways and emerging therapies. J Thyroid Res. 2011;2011:815826. doi: 10.4061/2011/815826

26. Yakes FM, Chen J, Tan J, Yamaguchi K, Shi Y, Yu P, etal. Cabozantinib (XL184), a novel MET and VEGFR2 inhibitor, simultaneously suppresses metastasis, angiogenesis, and tumor growth. Mol Cancer Ther. 2011;10(12):2298308. doi: 10.1158/1535-7163.MCT-11-0264

27. Gordon MS, Vogelzang NJ, Schoffski P, Daud A, Spira AI, O'Keeffe BA, et al. Activity of cabozantinib (XL184) in soft tissue and bone: Results of a phase II randomized discontinuation trial (RDT) in patients (pts) with advanced solid tumors. J Clin Oncol. 2011;29 (Suppl 15)3010-3010. doi: 10.1200/ jco.2011.29.15_suppl.3010

28. Al-Salama ZT, Keating GM. Cabozantinib: A Review in Advanced Renal Cell Carcinoma. Drugs. 2016;76(18):1771-8. doi: 10.1007/s40265-016-0661-5

29. Jimenez C, Waguespack S, Habra M, Busaidy N, Dadu R, Tamsen G, et al Cabozantanib in patients with unresectable metastatic pheochromocytoma and paraganglioma. Global Academic Programs of Cancer Centers Symposium; Houston, Tx2017.

30. Roman-Gonzalez A, Jimenez C. Malignant pheochromocytoma-paraganglioma: pathogenesis, TNM staging, and current clinical trials. Curr Opin Endocrinol Diabetes Obes. 2017;24(3):174-183. doi: 10.1097/ MED.0000000000000330

31. Cohen EE, Tortorici M, Kim S, Ingrosso A, Pithavala YK, Bycott P. A Phase II trial of axitinib in patients with various histologic subtypes of advanced thyroid cancer: long-term outcomes and pharmacokinetic/pharmacodynamic analyses. Cancer Chemother Pharmacol. 2014;74(6):1261-70. doi: 10.1007/s00280-014-2604-8

32. Frank-Raue K, Ganten M, Kreissl MC, Raue F. Rapid response to sorafenib in metastatic medullary thyroid carcinoma. Exp Clin Endocrinol Diabetes. 2011;119(3):151-5. doi: 10.1055/s-0030-1262836

33. Ravaud A, de la Fouchardière C, Asselineau J, Delord JP, Do Cao C, Niccoli P, et al. Efficacy of sunitinib in advanced medullary thyroid carcinoma: intermediate results of phase II THYSU. Oncologist. 2010;15(2):212-3; author reply 4. doi: 10.1634/theoncologist.2009-0303

34. van Geel RM, Beijnen JH, Schellens JH. Concise drug review: pazopanib and axitinib. Oncologist. 2012;17(8):1081-9. doi: 10.1634/theoncologist.2012-0055

35. Bass MB, Sherman SI, Schlumberger MJ, Davis MT, Kivman L, Khoo HM, et al. Biomarkers as predictors of response to treatment with motesanib in patients with progressive advanced thyroid cancer. J Clin Endocrinol Metab. 2010;95(11):5018-27. doi: 10.1210/jc.2010-0947
36. Sherman SI, Wirth LJ, Droz JP, Hofmann M, Bastholt L, Martins RG, et al. Motesanib diphosphate in progressive differentiated thyroid cancer. $\mathrm{N}$ Engl J Med. 2008;359(1):31-42. doi: 10.1056/NEJMoa075853

37. Kummar S, Lassen UN. TRK Inhibition: A New Tumor-Agnostic Treatment Strategy. Target Oncol. 2018;13(5):545-56. doi: 10.1007/s11523-0180590-1

38. Cocco E, Scaltriti M, Drilon A. NTRK fusion-positive cancers and TRK inhibitor therapy. Nat Rev Clin Oncol. 2018;15(12):731-47. doi: 10.1038/ s41571-018-0113-0

39. Scott LJ. Larotrectinib: First Global Approval. Drugs. 2019;79(2):201-6 doi: $10.1007 / s 40265-018-1044-x$

40. Laetsch TW, Hawkins DS. Larotrectinib for the treatment of TRK fusion solid tumors. Expert Rev Anticancer Ther. 2019;19(1):1-10. doi: 10.1080/14737140.2019.1538796

41. Drilon A, Laetsch TW, Kummar S, DuBois SG, Lassen UN, Demetri GD, et al. Efficacy of Larotrectinib in TRK Fusion-Positive Cancers in Adults and Children. N Engl J Med. 2018;378(8):731-9. doi: 10.1056/NEJMoa1714448

42. Berger S, Martens UM, Bochum S. Larotrectinib (LOXO-101). Recent Results Cancer Res. 2018;211:141-51. doi: 10.1007/978-3-319-91442-8 10

43. Laetsch TW, DuBois SG, Mascarenhas L, Turpin B, Federman N, Albert CM, et al. Larotrectinib for paediatric solid tumours harbouring NTRK gene fusions: phase 1 results from a multicentre, open-label, phase $1 / 2$ study. Lancet Oncol. 2018;19(5):705-14. doi: 10.1016/S1470-2045(18)30119-0

44. Hong DS, Bauer TM, Lee JJ, Dowlati A, Brose MS, Farago AF, et al. Larotrectinib in adult patients with solid tumours: a multicentre, open-label, phase I dose-escalation study. Ann Oncol. 2019;30(2):325-31. doi: 10.1093/annonc/mdy539

45. Sabari JK, Siau ED, Drilon A. Targeting RET-rearranged lung cancers with multikinase inhibitors. Oncoscience. 2017;4(3-4):23-4. doi: 10.18632/ oncoscience. 345

46. Wells SA, Gosnell JE, Gagel RF, Moley J, Pfister D, Sosa JA, et al. Vandetanib for the treatment of patients with locally advanced or metastatic hereditary medullary thyroid cancer. J Clin Oncol. 2010;28(5):767-72. doi: 10.1200/JCO.2009.23.6604

47. Karras S, Anagnostis P, Krassas GE. Vandetanib for the treatment of thyroid cancer: an update. Expert Opin Drug Metab Toxicol. 2014;10(3):46981. doi: $10.1517 / 17425255.2014 .885015$

48. BLU-667 Targets RET-Altered Cancers. Cancer Discov. 2018;8(6):0F8. doi 10.1158/2159-8290.CD-NB2018-050

49. Subbiah V, Gainor JF, Rahal R, Brubaker JD, Kim JL, Maynard M, et al. Precision Targeted Therapy with BLU-667 for RET-Driven Cancers. Cancer Discov. 2018;8(7):836-49. doi: 10.1158/2159-8290.CD-18-0338

50. Cabanillas ME, Ferrarotto R, Garden AS, Ahmed S, Busaidy NL, Dadu R, et al. Neoadjuvant BRAF- and Immune-Directed Therapy for Anaplastic Thyroid Carcinoma. Thyroid. 2018;28(7):945-51. doi: 10.1089/thy.2018.0060

51. Cabanillas ME, Patel A, Danysh BP, Dadu R, Kopetz S, Falchook G. BRAF Inhibitors: Experience in Thyroid Cancer and General Review of Toxicity. Horm Cancer. 2015;6(1):21-36. doi: 10.1007/s12672-014-0207-9

52. Hauschild A, Dummer R, Schadendorf D, Santinami M, Atkinson V, Mandalà $\mathrm{M}$, et al. Longer Follow-Up Confirms Relapse-Free Survival Benefit With Adjuvant Dabrafenib Plus Trametinib in Patients With Resected BRAF V600-Mutant Stage III Melanoma. J Clin Oncol. 2018;36(35):3441-3449. doi: 10.1200/JCO.18.01219

53. Odogwu L, Mathieu L, Blumenthal G, Larkins E, Goldberg KB, Griffin N, et al. FDA Approval Summary: Dabrafenib and Trametinib for the Treatment of Metastatic Non-Small Cell Lung Cancers Harboring. Oncologist. 2018;23(6):740-5. doi: 10.1634/theoncologist.2017-0642

54. Subbiah V, Kreitman RJ, Wainberg ZA, Cho JY, Schellens JHM, Soria JC, et al Dabrafenib and Trametinib Treatment in Patients With Locally Advanced or Metastatic BRAF V600-Mutant Anaplastic Thyroid Cancer. J Clin Oncol. 2018;36(1):7-13. doi: 10.1200/JCO.2017.73.6785

55. Dobosz T, Lukienczuk T, Sasiadek M, Kuczyńska A, Jankowska E, Blin N Microsatellite instability in thyroid papillary carcinoma and multinodular hyperplasia. Oncology. 2000;58(4):305-10. doi: 10.1159/000012117

56. Genutis LK, Tomsic J, Bundschuh R, Brock P, Williams MD, Roychowdhury $\mathrm{S}$, et al. Microsatellite Instability Occurs in a Subset of Follicular Thyroid Cancers. Thyroid. 2019;29(4):523-29. doi: 10.1089/thy.2018.0655

57. Naoum GE, Morkos M, Kim B, Arafat W. Novel targeted therapies and immunotherapy for advanced thyroid cancers. Mol Cancer. 2018;17(1):51. doi: 10.1186/s12943-018-0786-0

58. Weitzman SP, Sherman SI. Novel Drug Treatments of Progressive Radioiodine-Refractory Differentiated Thyroid Cancer. Endocrinol Metab Clin North Am. 2019;48(1):253-68. doi: 10.1016/j.ecl.2018.10.009

59. Antonelli A, Ferrari SM, Fallahi P. Current and future immunotherapies for thyroid cancer. Expert Rev Anticancer Ther. 2018;18(2):149-59. doi: $10.1080 / 14737140.2018 .1417845$ 


\section{Medtronic}

\section{BOMBA DE INSULINA MINIMED ${ }^{T M}$ 670G}

CONTECNOLOGÍA SMARTGUARD TM

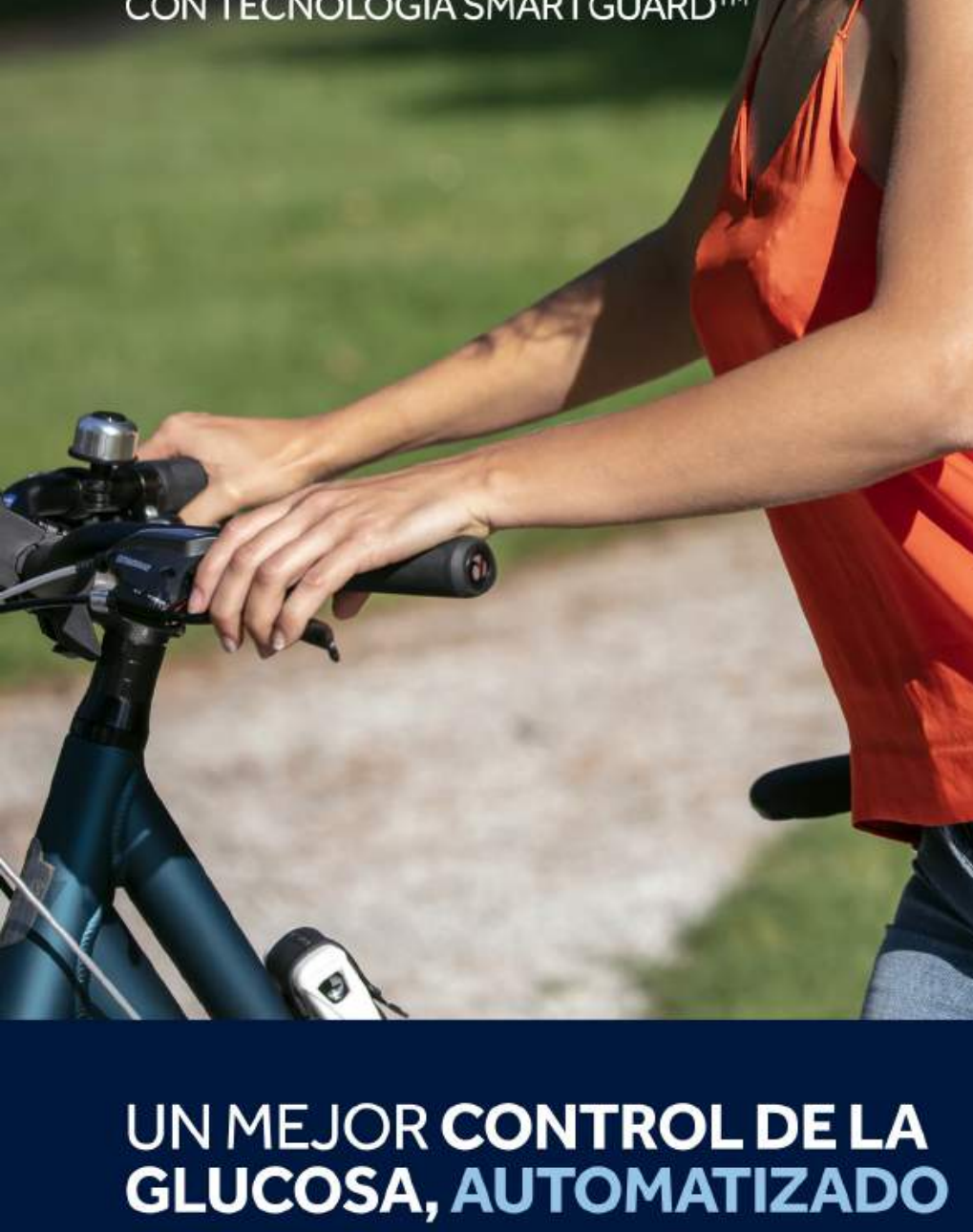

MÁS TIEMPO ENRANGO

Al automatizar la entrega de insulina, el sistema MiniMed ${ }^{\mathrm{TM}} 670 \mathrm{G}$ con tecnología SmartGuard, ayuda a incrementar el tiempo en rango del paciente..$^{1,2}$

\section{MEJOR CONTROL}

¿Cómo sería su día a día si los valores de glucosa de sus pacientes se mantuvieran entre 70 y $180 \mathrm{mg} /$ dL de dia y de noche, automáticamente?*

¡Ahora es posible!

\section{RESULTADOS CLÍNICOS $^{1,2}$}

- Reducción en HbA1c de 7,4 a 6,9

- Incremento en TIR de $66,7 \%$ a $72,2 \%$

- Cero casos de hipoglucemia grave**

\section{CARELINK SYSTEM}

Gestione y realice un seguimiento del tratamiento con bomba de insulina de sus pacientes, a través del software en linea que genera informes completose intuitivos.
Ajusta automáticamente la infusión de insulina basal según las lecturas de monitorización continua de glucosa (MCG), adaptándose a las necesidades únicas de insulina de cada paciente.*

\footnotetext{
**enla fase deestudio
} 